\title{
Advanced Neuroendocrine Neoplasm
}

National Cancer Institute

\section{Source}

National Cancer Institute. Advanced Neuroendocrine Neoplasm. NCI Thesaurus. Code C156486.

A neuroendocrine neoplasm that has spread extensively to other anatomic sites or is no longer responding to treatment. 